\title{
EDITORIAL
}

\section{VAccine Therapy of Gonococcal Infections}

IN this and the April number of this Journal we have given considerable space to the discussion on vaccine therapy of gonococcal infections which was held at meetings of the Medical Society for the Study of Venereal Diseases on January 23rd and February 27th.

The limitations of the frontal attack on the gonococcus are well known, and the desire for a means of reaching and destroying it from the rear is felt by every one interested in the treatment of gonorrhœa.

To the student of gonococcal infections it must be clear that the patient depends for his recovery on his resistance to the micro-organism; otherwise how would we explain the variable course of the disease in different patients under the same kinds of local and general treatment? For example, most workers have experience of patients in whom an urethritis with profuse discharge containing gonococci persisted unabated far beyond the average time, in spite of careful local treatment, and yet of others who recovered in a comparatively few days, though the treatment was on lines which our general experience has led us to believe are bad. The belief that resistance of the tissues to the parasite is a potent factor in the prognosis has for natural consequence the desire to raise this resistance to the utmost, and in our search for agents to this end we turn almost automatically to vaccines, encouraged thereto by the success which this form of therapy has achieved in other bacterial infections.

So far, good! But here we meet with a check which is tantalising. For, while we cannot believe that the numerous workers who have reported good results from the vaccine therapy of gonococcal infections have been mistaken in their observations and conclusions, we must confess that often vaccines have proved disappointing, so that many express themselves as sceptical. At such a check it was well to go carefully over the ground, as was done in the discussion, in the hope that ways of discovering 


\section{BRITISH JOURNAL OF VENEREAL DISEASES}

and eliminating the causes of failure might be suggested. In this respect the discussion was fruitful, and our readers will find in it much that is corrective of error as well as a stimulus to further investigation which may show us how to extract the best from a form of treatment which, theoretically at least, should be helpful in a disease which does not respond too well to ordinary methods.

Considering the question first from the point of view of the antigen, there was a concensus of opinion that gonococci at their best appear to be indifferent antigens, and that such antigenic value as they possess diminishes in proportion to the time they have been under artificial conditions; hence it is agreed that we should employ emulsions made only from germs which have been isolated recently. It appears, also, that an important cause of failure may lie in the multiplicity of strains of gonococci. The common practice at present is to employ in stock gonococcal vaccines a number of cultures of organisms obtained from different sources. The practice is described as using many strains, but the differentiation of strains is a lengthy and complicated process which is ordinarily not carried out in the making of gonococcal vaccines, and, in fact, one vaccine made of germs derived from a dozen sources might, for all we know, contain only one strain, while the next might contain a dozen. To increase the uncertainty, we do not know clearly if all strains are equally powerful antigens against homologous infections, and feeble only against heterologous, or some are good antigens also against certain heterologous strains, or, again, if some are good and some indifferent against homologous strains. To determine these questions, and especially to discover ways of detecting the good antigens very quickly after their isolation, considerably more research is necessary. On the result depends the choice of vaccine for the individual patient. If it should prove to be the case that the immunising power of a gonococcal vaccine is always good in an infection with its homologous strain, but poor against heterologous infections, clearly it would be illogical to give a vaccine made up of a number of strains of which only one, that is, a fraction of the dose, is specific. Our only method at present of assuring the administration of an homologous vaccine is to prepare one from gonococci isolated from the patient, a method for which Dr. Eyre expressed a decided prefer- 


\section{GONOCOCCAL INFECTIONS}

ence, but one which is practicable only for the few. To ensure homologous vaccines for the multitude we should require a means of identifying rapidly the patient's gonococcus with one of the strains we have isolated already and made into vaccine. Perhaps the simultaneous application of a number of cutaneous tests, employing one strain in each, would provide the necessary key.

It would be fortunate if further research were to show that one or a few strains immunise powerfully against allothers, and also if a means of identifying the good immunisers rapidly could be discovered. Dr. Eyre's view is that gonococci isolated from the eye are poor antigens, and those from joints better, while the best are to be found amongst those isolated from acute infections of the genital passages, and certainly there is some clinical evidence to support the impression that gonococci vary in their antigenic value, since good results from stock vaccine treatment seem to occur in batches which are followed by periods when results are indifferent or poor.

On the treatment of the germ chosen for the vaccine the discussion disclosed a sharp difference of opinion. Some are convinced of the value of detoxication, originated by Dr. D. Thomson, while others are doubtful; may the reason be that those happened to administer an homologous detoxicated vaccine, or one of generally good immunising value, while the doubters have happened to use a heterologous strain, or one which is naturally a poor antigen ? It seems to us that until the influence of strain on the immunity response is thoroughly worked out, other questions must remain undecided.

Whilst so little is known about the influence of strains and of methods of preparation, it is not surprising that there should be so much difference of practice in the matter of dosage. As Dr. Eyre pointed out, a vaccine made from organisms which have been isolated recently cannot be administered in such large doses as one made from gonococci which have been for months under artificial conditions. Here is especially the need for collaboration between the clinician and the pathologist. Usually the clinician does not know if the vaccine is made from year-old or day-old gonococci, or if it was heated or killed only by antiseptics. It would be better if the clinician could always be told something of the origin 


\section{BRITISH JOURNAL OF VENEREAL DISEASES}

of the vaccine and of its treatment, and if the clinician were to tell the pathologist about the effects of different doses on his patients. At present the administration of gonococcal vaccine follows too mechanically programmes suggested in books, or by pathologists who have themselves had little guidance from information as to the effect of given doses of the vaccines they have supplied on given types of case.

Besides the question of dosage and intervals between doses is what may prove to be the important one of the site of inoculation, which was raised by Dr. Panton. Hitherto the main discussion between workers on this point has been between subcutaneous, or intramuscular, and intravenous, each of which has its strong advocates. Dr. Panton voiced the possibility of better results from intracutaneous inoculation, a method which has support in the finding of E. F. Müller (Münch. med. Wchnschr., January 2nd, I920, p. 9) that the intracutaneous injection of "aolan " was followed by an urethral reaction in the form of irritation and discharge, an effect which was produced only by a considerably larger dose of the same substance given subcutaneously.

For the success of vaccine therapy it is necessary, also, that the patient's tissues should be in the best condition to respond to the antigen. On the question of stimulating response, nothing was said of the possible use of endocrines, but on the value of shock therapy employed simultaneously with specific vaccines the views of the clinicians who took part in this discussion seem to have been fairly well agreed. Perhaps, however, more might have been said on the dosage of the substances used in this adjuvant form of treatment. That it is valuable we do not doubt, but we believe that it can be over-done and that the dosage should be regulated with as much nicety as should be that of the specific vaccine.

Briefly, we think that the discussion on gonococcal vaccine therapy has been useful in showing the lines on which valuable work can be done. Nobody can say that vaccines are useless in gonococcal infections; there is too much evidence to the contrary. Everybody must admit, however, that we have much to learn before we can rely with reasonable assurance on obtaining satisfactory results. If we may repeat a suggestion already offered in these remarks, we would say that the most 


\section{GONOCOCCAL INFECTIONS}

hopeful lines of future work are in first finding the influence of strain on immunising power, and then a method of discovering rapidly the right strain for the individual patient. After this can be determined the best route of introduction and the dosage.

For the acquisition of this knowledge an immense amount of research is necessary, and in it is required the closest collaboration between clinicians and pathologists. We suggest that it is work which is worth while. Gonorrhœa does not appear to kill so many people, perhaps, as does typhoid fever, but it certainly afflicts very many more and accounts for much more suffering, so that the improvement of gonococcal vaccine deserves at least the same amount of research as has been devoted to antityphoid vaccine. While we have hoped in this summary to stimulate pathologists already engaged in gonococcal work to investigate further on some such lines as we have indicated, we confess to some fear that it is work in which little progress can be made by those engaged also in routine clinical pathology. The gonococcus is not an easy subject for research; it does not grow very easily, it degenerates rapidly in culture, its anti-bodies are difficult to demonstrate, and the ordinary animals employed in experimental research are useless for its investigation. For these reasons, each step can be made only by infinite labour, and that by workers who can give to the research several uninterrupted hours a day. We could hope that some one able to do so would choose the supporting of such a research as his method of exercising a real philanthropy.

\section{NEURO-RECURRENCES FOLLOWING ARSENOBENZOL TREAATMENT}

In this number we publish a further contribution to the investigation of the Medical Society for the Study of Venereal Diseases into the incidence of clinical neurorecurrences in syphilitic patients who have, prior to the onset of the nerve symptoms, been treated with arsenobenzol. Mr. Nicol's search leaves us still with no evidence that intensive arsenobenzol treatment hastens the onset, or increases the incidence, of neuro-recurrences. Indeed, it seems so far to point to an opposite conclusion. We would again ask our readers to do their best to cause all 


\section{BRITISH JOURNAL OF VENEREAL DISEASES}

the evidence possible to be brought forward. Medical officers of venereal diseases treatment centres who have not yet answered the questionnaire issued by the M.S.S.V.D. will help the investigation by doing so, as also by stimulating their colleagues in neurological clinics to send us particulars of their experience. The question is of world-wide interest, and we invite also the contributions of readers in other countries.

\section{SIR HUMPHRY DAVY ROLLESTON}

It is peculiarly appropriate that the first expression of congratulatory goodwill to appear in this Journal should be extended to Sir Humphry Davy Rolleston. He gave sage advice and timely encouragement as a pioneer member of the Editorial Committee, to which body he still belongs. We ask his acceptance of our official and personal wishes that he may enjoy a long and successful tenure of the honourable and ancient office of the Regius Professor of Physic in the University of Cambridge. 